\title{
Pseudo orbit tracing property and fixed points
}

\author{
by Masatoshi OKa (Tokyo)
}

\begin{abstract}
If a continuous map $f$ of a compact metric space has the pseudo orbit tracing property and is $h$-expansive then the set of all fixed points of $f$ is totally disconnected.
\end{abstract}

The pseudo orbit tracing property is one of the most important notions in dynamical systems. The observation of periodic points is the first step to know the orbit structure of a system. In this paper we investigate the sets of all fixed points of continuous maps with the pseudo orbit tracing property. Our result gives a partial positive answer to the following question by Morimoto [8]:

Is the set of all fixed points of a $C^{1}$ diffeomorphism of a compact manifold totally disconnected if the diffeomorphism has the pseudo orbit tracing property?

We show in this paper that the set of all fixed points of a continuous map which has the pseudo orbit tracing property is totally disconnected under a supplementary condition of expansiveness with respect to entropy.

Let $X$ be a compact metric space with metric $d$ and $f: X \rightarrow X$ be a continuous map. For $\delta>0$ a sequence $\left\{x_{i}\right\}$ of points in $X$ is said to be a $\delta$-pseudo orbit if $d\left(f\left(x_{i}\right), x_{i+1}\right)<\delta$ for $i=0,1,2, \ldots$ We say that $f$ has the pseudo orbit tracing property if for any $\varepsilon>0$ there is $\delta>0$ such that for any $\delta$-pseudo orbit $\left\{x_{i}\right\}$ there is $y \in X$ satisfying $d\left(f^{i}(y), x_{i}\right)<\varepsilon$ for any $i \geq 0$. The $y$ is said to be an $\varepsilon$-tracing point of $\left\{x_{i}\right\}$. For $\varepsilon>0$ and $x \in X$ we denote by $\Phi_{\varepsilon}(x)$ the set $\bigcap_{n>0} f^{-n} B_{\varepsilon}\left(f^{n}(x)\right)$, where $B_{\varepsilon}(x)=\{y \in X: d(y, x) \leq \varepsilon\}$. We say that a subset $E$ of $X(n, \delta)$-spans a subset $K$ if for each $y \in K$ there is $x \in E$ such that $d\left(f^{i}(x), f^{i}(y)\right) \leq \delta$ for all $i \in[0, n)$. Let $\gamma_{n}(\delta, K)$ be the minimum cardinality of sets which $(n, \delta)$-span $K$. Since $\Phi_{\varepsilon}(x)$ is compact, $\gamma_{n}\left(\delta, \Phi_{\varepsilon}(x)\right)$ is finite. Define

1991 Mathematics Subject Classification: Primary 58F25.

Key words and phrases: pseudo orbit tracing property, $h$-expansive. 


$$
\begin{aligned}
\bar{\gamma}_{f}\left(\delta, \Phi_{\varepsilon}(x)\right) & =\limsup _{n \rightarrow \infty} \frac{1}{n} \log \gamma_{n}\left(\delta, \Phi_{\varepsilon}(x)\right), \\
h\left(f, \Phi_{\varepsilon}(x)\right) & =\lim _{\delta \rightarrow 0} \bar{\gamma}_{f}\left(\delta, \Phi_{\varepsilon}(x)\right) .
\end{aligned}
$$

Then $\bar{\gamma}_{f}\left(\delta, \Phi_{\varepsilon}(x)\right)$ increases as $\delta$ decreases. We denote by $\operatorname{Fix}(f)$ the set $\{x \in X: f(x)=x\}$. Our result is as follows.

Theorem. Let $f$ have the pseudo orbit tracing property. If for any $x \in \operatorname{Fix}(f)$ there is $\varepsilon>0$ with $h\left(f, \Phi_{\varepsilon}(x)\right)=0$, then $\operatorname{Fix}(f)$ is totally disconnected.

A continuous map $f: X \rightarrow X$ is called $h$-expansive if there exists $\varepsilon>0$ such that $h\left(f, \Phi_{\varepsilon}(x)\right)=0$ for any $x \in X$ (cf. [2]).

COROLlary. If an h-expansive map $f$ has the pseudo orbit tracing property, then $\operatorname{Fix}(f)$ is totally disconnected.

Misiurewicz constructed a $C^{1}$ diffeomorphism which is not $h$-expansive (cf. [7]). Thus Morimoto's question is still open for $C^{1}$ diffeomorphisms.

To prove the Theorem we need two lemmas. For $x, y \in X$, a $\delta$-chain from $x$ to $y$ is a finite $\delta$-pseudo orbit $\left\{x_{0}, x_{1}, \ldots, x_{m}\right\}$ with $x_{0}=x, x_{m}=y$. The number $m+1$ is called the length of the chain.

Lemma 1. If a closed subset $C$ of $X$ is connected, then for any $\alpha>0$ there is a positive integer $m$ such that for any $x, y \in C$ there is an $\alpha$-chain from $x$ to $y$ with length at most $m+1$.

Proof. Let $U_{\alpha / 2}(x)=\{y \in X: d(y, x)<\alpha / 2\}$. Since $C$ is compact, we can find $x_{1}, \ldots, x_{k} \in C$ such that $\bigcup_{i=1}^{k} U_{\alpha / 2}\left(x_{i}\right) \supset C$. Put $m=k+1$. Since $C$ is connected, for any $p, q \in\left\{x_{1}, \ldots, x_{k}\right\}$ we take $x_{i_{0}}, \ldots, x_{i_{l}} \in$ $\left\{x_{1}, \ldots, x_{k}\right\}$ with $x_{i_{0}}=p, x_{i_{l}}=q$ such that $U_{\alpha / 2}\left(x_{i_{j}}\right) \cap U_{\alpha / 2}\left(x_{i_{j+1}}\right) \neq \emptyset$ for $j=0,1, \ldots, l-1$. Thus $\left\{p, x_{i_{1}}, \ldots, x_{i_{l-1}}, q\right\}$ is an $\alpha$-chain in $C$. We can assume that $x_{i_{s}} \neq x_{i_{t}}$ for $s \neq t$ (otherwise we replace the $\alpha$-chain by one with smaller $l$ ). Hence the length of $\left\{p, x_{i_{1}}, \ldots, x_{i_{l-1}}, q\right\}$ is at most $k$. For any $x, y \in C$, we can find $p, q \in C$ such that $U_{\alpha / 2}(p) \ni x, U_{\alpha / 2}(q) \ni y$ and so the $\alpha$-chain $\left\{x, p, x_{i_{1}}, \ldots, x_{i_{l-1}}, q, y\right\}$ has length at most $k+2=m+1$.

The following is an immediate fact from general topology.

Lemma 2. Let $F$ be a closed subset of $X$. If for each $x \in F$ there is $\varepsilon>0$ such that $B_{\varepsilon}(x) \cap F$ is totally disconnected, then so is $F$.

The proof is omitted. See [6] for the details.

Proof of Theorem. Put $F=\operatorname{Fix}(f)$. Since $F$ is a closed subset of $X$, by Lemma 2 it is sufficient to show that for any $x \in F$ there is $\varepsilon>0$ such that $B_{\varepsilon / 2}(x) \cap F$ is totally disconnected. 
Let $x \in F$ and assume that $h\left(f, \Phi_{\varepsilon}(x)\right)=0$ for some $\varepsilon>0$. Let $C$ denote the connected component of $B_{\varepsilon / 2}(x) \cap F$ containing $x$ and assume that $C$ is not a one-point set. Then $C$ is not finite and so for any positive integer $a$ we can take $p_{1}, \ldots, p_{a} \in C\left(p_{i} \neq p_{j}\right.$ if $\left.i \neq j\right)$. Take $\varepsilon_{0}$ with $0<\varepsilon_{0}<\min \left\{d\left(p_{i}, p_{j}\right): 1 \leq i \neq j \leq a\right\}$. Since $f$ has the pseudo orbit tracing property, for any $\mu$ with $0<\mu<\min \left\{\varepsilon_{0} / 6, \varepsilon / 2\right\}$ there is $\alpha$ with $0<\alpha<\min \left\{\varepsilon_{0} / 6, \varepsilon / 2\right\}$ such that any $\alpha$-pseudo orbit of $f$ is $\mu$-traced by a point $y \in X$. By Lemma 1 we can find a positive integer $m$ such that for any $\bar{x}, \bar{y} \in C$ there is an $\alpha$-chain $\left\{x_{0}, \ldots, x_{m}\right\} \subset C$ from $\bar{x}$ to $\bar{y}$ with length at most $m+1$.

Take an $n$-tuple $\left(z_{0}, \ldots, z_{n-1}\right)$ such that $z_{j}=p_{i_{j}} \in\left\{p_{1}, \ldots, p_{a}\right\}$ for $j=0, \ldots, n-1$. Then for each consecutive pair $z_{i}, z_{i+1}(i=0, \ldots, n-2)$ we can find an $\alpha$-chain in $C$ from $z_{i}$ to $z_{i+1}$ with length at most $m+1$. Since $C$ is connected the $\alpha$-chains may be assumed to have length $m+1$ by adding some points of $C$ if necessary (cf. [9]). Hence if $\left\{x_{0}^{(i)}, x_{1}^{(i)}, \ldots, x_{m}^{(i)}\right\}$ denotes the $\alpha$-chain for the pair $z_{i}, z_{i+1}$, where $x_{0}^{(i)}=z_{i}$ and $x_{m}^{(i)}=z_{i+1}$, then $\left\{x_{0}^{(0)}, \ldots, x_{m-1}^{(0)}, x_{0}^{(1)}, x_{1}^{(1)}, \ldots, x_{m-1}^{(1)}, x_{0}^{(2)}, \ldots, x_{m-1}^{(n-2)}, x_{m}^{(n-2)}, \ldots\right\}$ is an $\alpha$-pseudo orbit of $f$ such that $x_{m}^{(k)}=x_{0}^{(k+1)}$ for $k=0,1, \ldots, n-3$. Since $f$ has the pseudo orbit tracing property there is $u \in X$ such that

$$
d\left(f^{l m+j}(u), x_{j}^{(l)}\right)<\mu
$$

for $l=0, \ldots, n-2$ and $j=0, \ldots, m$. Thus $u \in \Phi_{\varepsilon}(x)$ because $x_{j}^{l} \in C \subset$ $B_{\varepsilon / 2}(x)$ and $\mu<\varepsilon / 2$. Take $0<\delta<\varepsilon_{0} / 6$ and an $(m n, \delta)$-spanning set $E$ for $\Phi_{\varepsilon}(x)$. Then we can find $\bar{u} \in E$ such that

$$
d\left(f^{k}(\bar{u}), f^{k}(u)\right) \leq \delta
$$

for $k=0,1, \ldots, m n-1$. If we take another $n$-tuple $\left(z_{0}^{\prime}, \ldots, z_{n-1}^{\prime}\right)$ from the set $\left\{p_{1}, \ldots, p_{a}\right\}$, then there are $v \in \Phi_{\varepsilon}(x)$ and $\bar{v} \in E$ such that

$$
d\left(f^{l m+j}(v), x_{j}^{(l)}\right)<\mu
$$

for $l=0, \ldots, n-2$ and $j=0, \ldots, m$, and

$$
d\left(f^{k}(\bar{v}), f^{k}(v)\right) \leq \delta
$$

for $k=0,1, \ldots, m n-1$. We have $u \neq v$. For if $\left(z_{0}, z_{1}, \ldots, z_{n-1}\right) \neq\left(z_{0}^{\prime}, z_{1}^{\prime}, \ldots\right.$ $\left.\ldots, z_{n-1}^{\prime}\right)$, then $z_{l} \neq z_{l}^{\prime}$ for some $l$. Thus

$$
\begin{aligned}
\varepsilon_{0}<d\left(z_{l}, z_{l}^{\prime}\right) & \leq d\left(z_{l}, f^{l m}(u)\right)+d\left(f^{l m}(u), f^{l m}(v)\right)+d\left(f^{l m}(v), z_{l}^{\prime}\right) \\
& <\mu+d\left(f^{l m}(u), f^{l m}(v)\right)+\mu .
\end{aligned}
$$

Hence $d\left(f^{l m}(u), f^{l m}(v)\right)>\varepsilon_{0}-2 \mu>2 \varepsilon_{0} / 3>0$ and so $u \neq v$. We also have $\bar{u} \neq \bar{v}$. Indeed, if $z_{l} \neq z_{l}^{\prime}$, then $d\left(f^{l m}(u), z_{l}\right)<\mu$ and $d\left(f^{l m}(v), z_{l}^{\prime}\right)<\mu$ by 
(1) and (3). Hence

$$
\begin{aligned}
\varepsilon_{0}< & d\left(z_{l}, z_{l}^{\prime}\right) \\
\leq & d\left(z_{l}, f^{l m}(u)\right)+d\left(f^{l m}(u), f^{l m}(\bar{u})\right)+d\left(f^{l m}(\bar{u}), f^{l m}(\bar{v})\right) \\
& +d\left(f^{l m}(\bar{v}), f^{l m}(v)\right)+d\left(f^{l m}(v), z_{l}^{\prime}\right) \\
\leq & \mu+\delta+d\left(f^{l m}(\bar{u}), f^{l m}(\bar{v})\right)+\delta+\mu \\
\leq & 2(\mu+\delta)+d\left(f^{l m}(\bar{u}), f^{l m}(\bar{v})\right) \\
\leq & 2 \varepsilon_{0} / 3+d\left(f^{l m}(\bar{u}), f^{l m}(\bar{v})\right)
\end{aligned}
$$

and so $d\left(f^{l m}(\bar{u}), f^{l m}(\bar{v})\right)>\varepsilon_{0} / 3>0$. Hence $\bar{u} \neq \bar{v}$. Therefore we have $\operatorname{Card}(E) \geq a^{n}$, where $\operatorname{Card}(E)$ denotes the cardinality of the set $E$. Thus $\bar{\gamma}_{f}\left(\delta, \Phi_{\varepsilon}(x)\right) \geq \frac{1}{m} \log a$ and so $h\left(f, \Phi_{\varepsilon}(x)\right) \geq \frac{1}{m} \log a>0$, which is a contradiction. Thus $C$ is a one-point set and $\operatorname{Fix}(f)$ is totally disconnected.

\section{References}

[1] R. L. Adler, A. G. Konheim and M. H. McAndrew, Topological entropy, Trans. Amer. Math. Soc. 114 (1965), 309-319.

[2] R. Bowen, Entropy-expansive maps, ibid. 164 (1972), 323-331.

[3] - Entropy for group endomorphisms and homogeneous spaces, ibid. 153 (1971), 401-414.

[4] M. Datey a ma, Homeomorphisms with the pseudo orbit tracing property of the Cantor set, Tokyo J. Math. 6 (1983), 287-290.

[5] M. Denker, C. Grillenberger and K. Sigmund, Ergodic Theory on Compact Spaces, Lecture Notes in Math. 527, Springer, 1976.

[6] W. Hurewicz and H. Wallman, Dimension Theory, Princeton University Press, 1948.

[7] M. Misiurewicz, Diffeomorphisms without any measure with maximal entropy, Bull. Acad. Polon. Sci. 21 (1973), 903-910.

[8] A. Morimoto, The method of pseudo-orbit tracing and stability of dynamical systems, Seminar note 39, University of Tokyo, 1979 (in Japanese).

[9] T. Shimomura, On a structure of discrete dynamical systems from the view point of chain components and some applications, Japan. J. Math. 15 (1989), 99-126.

DEPARTMENT OF MATHEMATICS SCIENCE UNIVERSITY OF TOKYO NODA-SHI, CHIBA-KEN 278 JAPAN 